REJ - Revista de Estudios de la Justicia - No 4 - Año 2004

\title{
CONSIDERACIONES SOBRE LA PRUEBA DEL DOLO
}

Ramón Ragués i Vallès*

\begin{abstract}
I. Introducción; II. Concepto de dolo: breve panorámica; III. La prueba de los hechos subjetivos en el proceso; IV. Propuesta de tratamiento: las reglas de atribución del conocimiento; V. La prueba del dolo en los delitos de resultado.
\end{abstract}

\section{INTRODUCCIÓN}

Para resolver la cuestión de cómo se prueba el dolo en el proceso penal es imprescindible contar con dos herramientas teóricas: una teoría del dolo y una teoría de la prueba. La teoría del dolo hace falta porque, sin saber qué es aquello que debe ser probado, difícilmente se puede decidir cómo ha de llevarse a cabo la actividad probatoria en cuestión. Y, en segundo lugar, tampoco cabe prescindir de la teoría de la prueba, pues sin ella no es posible instruir al operador jurídico que se encuentra ante un caso concreto sobre cómo y cuándo debe dar por acreditada la presencia de aquellos elementos fácticos que permiten afirmar el concepto cuya eventual aplicación se plantea.

Responder con un mínimo fundamento a la pregunta de qué es el dolo requiere un grado de argumentación que excede con mucho del espacio aquí disponible. No en vano, la definición de dolo es una de las materias que, ya desde el siglo XIX, mayor controversia ha suscitado entre los penalistas que se han ocupado de la teoría del delito. Sin embargo, dado que no es posible pasar a la segunda cuestión (“¿cómo se prueba el dolo?”) sin dar antes respuesta a la primera, a continuación se expondrá de forma muy breve cuál es el concepto de dolo del que se parte, justificando sucintamente semejante elección ${ }^{1}$. Acto seguido pasará a exponerse el tema central del presente trabajo: la prueba del dolo.

\section{CONCEPTO DE DOLO: BREVE PANORÁMICA}

Aunque tradicionalmente el dolo se ha definido como conciencia y voluntad de la realización de una conducta objetivamente típica, esta definición ha sido paulatinamente abandonada por la doctrina y por los tribunales, hasta el punto de poderse afirmar que, hoy en día, el dolo se concibe (de forma explícita o implícita) sólo como conciencia de la realización de un comportamiento típico objetivo. Por expresarlo de forma simple pero contundente, el dolo ya no es conocimiento y voluntad, sino únicamente conocimiento. A este cambio conceptual se ha llegado por diversas razones.

El motivo principal es que una aplicación coherente del dolo definido como voluntad o intención lleva a castigar como meras imprudencias supuestos que, ante los ojos de cualquier espectador, parecen mucho más cercanos al merecimiento de pena propio de los comportamientos dolosos que al de los negligentes. Esta valoración se

\footnotetext{
1 Para una fundamentación mucho más detallada del concepto de dolo que se defiende cfr. RAGUÉS I VALLÈS, El dolo y su prueba en el proceso penal, Barcelona, 1999, pp. 25-186. En las páginas de este trabajo puede encontrarse citada la principal bibliografía existente sobre la materia.
} 
Ragués - Consideraciones sobre la prueba del dolo

aprecia ya en el siglo XIX, cuando surgen en la doctrina figuras como el dolo directo de segundo grado o el dolo eventual, que no son otra cosa que ampliaciones del concepto de dolo pensadas para no tener que castigar como simples imprudencias supuestos que parecen mucho más graves. En este sentido puede afirmarse incluso que la idea del dolo como intención, proclamada en su momento por Feuerbach, nunca llegaría realmente a aplicarse, pues ya desde un primer momento se advirtió que era un concepto demasiado limitado ${ }^{2}$.

Esta necesidad de ampliar el concepto de dolo ha sido afrontada por la doctrina y la jurisprudencia recurriendo fundamentalmente a dos grandes métodos ${ }^{3}$ :

A) Un primer grupo de autores y jueces parte del principio apriorístico de que sólo la realización voluntaria o intencionada del tipo merece las penas previstas para los delitos dolosos $\mathrm{y}$, consecuentemente, trata de buscar dicho elemento en todos aquellos casos que le parecen merecedores de la pena del dolo. Tradicionalmente se ha denominado a este punto de vista como la teoría de la voluntad ${ }^{4}$.

B) Otro sector doctrinal prescinde de definiciones apriorísticas y analiza simplemente qué hechos considera acreedores de las penas previstas para las modalidades dolosas, buscando después denominadores comunes entre todos ellos que permitan construir una definición generalmente válida ${ }^{5}$.

En la práctica se ha demostrado que sólo el segundo método conduce a resultados satisfactorios. De hecho, la negativa de los partidarios del primer método a prescindir de un elemento volitivo les obliga a encontrar tal elemento en sucesos en que muy difícilmente lo hallaría cualquier ciudadano medio empleando el vocabulario cotidiano y, así, se acaba afirmando que quien simplemente acepta, se conforma o se resigna con la realización del tipo jurídicamente quiere dicha realización y, por tanto, actúa dolosamente ${ }^{6}$. En la práctica estos requisitos pseudovolitivos, como el "aceptar", "conformarse" o "resignarse", se deducen, sobre todo, de la circunstancia de que un

\footnotetext{
${ }^{2}$ La doctrina suele asociar el origen del concepto moderno de dolo a la figura de Feuerbach (y a su trabajo "Betrachtungen über dolus und culpa überhaupt und den dolus indirectus insbesondere" de 1804), por tratarse del primer autor que explícitamente rechazó las teorías objetivizantes del dolus indirectus y el dolus ex re que habían predominado hasta entonces, definiendo el dolo como intención entendida en términos exclusivamente psíquicos. Sobre este importante cambio cfr. LESCH, "Dolus directus, indirectus und eventualis", $J A, 1997$, p. 802 ss y PuPpe, $N K, \int 15$, n. ${ }^{\circ}$ marg. 22.

${ }^{3} \mathrm{La}$ discusión entre los partidarios de estos dos puntos de vista a menudo se ha contemplado como un debate circunscrito a la figura del dolo eventual. Sin embargo, dado que el dolo eventual marca la "frontera inferior" del concepto de dolo, en realidad esta discusión afecta a la globalidad de este concepto. En este sentido, FrISCH, Vorsatz und Risiko, Colonia, 1983, pp. 496 ss.

${ }^{4}$ Para una perspectiva de los autores partidarios de este planteamiento cfr. RAGUÉs I VALLÈs, El dolo..., op. cit., pp. 60-66 y pp. $103-105$.

${ }^{5}$ Una panorámica de los trabajos en los que se defiende esta perspectiva en RAGUÉS I VALLÈs, El dolo..., op. cit., pp. 66-71 y 88-97.

${ }^{6}$ Históricamente, estos planteamientos son herederos de la denominada "teoría del consentimiento" creada en Alemania en el siglo XIX y defendida por autores como Robert von Hippel, que la complementaban con la denominada fórmula de Frank, según la cual un sujeto consentía en la producción del resultado cuando podía afirmarse que habría actuado igualmente de haber sabido con seguridad que dicho resultado se iba a producir. Esta teoría — durante muchas décadas mayoritaria tanto en Alemania como en España - entra en crisis a partir de la década de 1950 con la sentencia del BGH alemán (BGHSt, vol. 7, p. 363 ss) sobre el denominado "caso de cinturón" (Lederriemenfall), aunque es posible todavía encontrarla en algunas resoluciones dictadas por el Tribunal Supremo español en la década de 1980.
} 
sujeto no haya desistido de su conducta pese a ser consciente del riesgo de realización típica que conllevaba.

Esta deducción automática del elemento pretendidamente volitivo a partir de la "actuación pese al conocimiento" plantea serias dudas sobre la necesidad conceptual de tal requisito, que, definido en estos términos, carece de un contenido propio que justifique su existencia ${ }^{7}$. Por otra parte, semejante elemento no desempeña papel relevante alguno en los delitos de mera actividad, donde incluso los partidarios del dolo como intención afirman la existencia de un delito doloso en casos de mero conocimiento de las circunstancias típicas: ¿acaso algún juez negaría el dolo del acusado de abusos sexuales que afirmara haber realizado tocamientos a una víctima de diez años conociendo perfectamente su edad pero "no habiendo querido hacerlo"? ¿o absolvería al conductor ebrio que declarase haber sido perfectamente consciente de que conducía bajo la influencia del alcohol pero haber realizado a disgusto dicha conducta?8. La idea del dolo como voluntad se enuncia pensando exclusivamente en los delitos de resultado, una circunstancia que en modo alguno puede juzgarse positivamente si lo que se pretende es construir una definición de dolo generalmente válida para toda la teoría del delito.

La afirmación de que para el dolo basta con el conocimiento ha sido, precisamente, la conclusión de la mayoría de partidarios del segundo método?. Por tal razón suele hacerse referencia a ellos con la denominación de teorías del conocimiento o de la representación. Según estos autores, para afirmar que se ha obrado dolosamente basta con acreditar que el sujeto activo ha actuado representándose la concurrencia en su conducta de los elementos objetivos exigidos por el tipo. En los delitos de resultado, esta exigencia se concreta entendiendo que, para afirmar el dolo, basta con que el sujeto haya obrado con conocimiento del riesgo concreto de producción del resultado ${ }^{10}$. En cambio, en los

\footnotetext{
${ }^{7}$ De entre las numerosas críticas que recibe este punto de vista merece la pena citar, aunque sólo sea por su plasticidad, las formuladas por HERZBERG, "Das Wollen beim Vorsatzdelikt und dessen Unterscheidung vom bewußt fahrlässigen Verhalten”, JZ, 1988, p. 575. Cfr. asimismo la crítica de KINDHÄUSER, "Der Vorsatz als Zurechnungskriterium", ZStW, 96 (1984), pp. 23-24 y, más recientemente, SCHÜNEMANN, "Vom philologischen zum typologischen Vorsatzbegriff”, Hirsch-FS, Berlín, 1999, p. 367.

${ }^{8}$ Cuestiona la necesidad del elemento volitivo en los delitos de mera actividad, entre otros, SILVA SÁNCHEZ, Aproximación al Derecho penal contemporáneo, Barcelona, 1992, pp. 401-402. Cfr. asimismo FrIsCH, Vorsatz, p. 381.

9 Algún autor como ENGISCH, en su Untersuchungen über Vorsatz und Fabrlässigkeit, Berlín, 1930 (reimp. 1995), pese a emplear este método llega a conclusiones distintas, como la afirmación de que para el dolo es necesaria, cuando menos, indiferencia. Sin embargo, su planteamiento no se ha impuesto. Aparentemente también emplean este método los autores que definen el dolo como "decisión contra el bien jurídico" o "asunción de los elementos constitutivos del injusto" (así, Hassemer o Schroth, respectivamente). Sin embargo, en el desarrollo de este planteamiento se constata un cierto apriorismo cuando se afirma que, para que concurra tal decisión o asunción, es imprescindible la presencia de algún elemento volitivo o pseudovolitivo. Cfr. en este sentido HASSEMER, "Kennzeichen des Vorsatzes", Armin Kaufmann-GS, Colonia, 1989, pp. 289309 [trad. M.M. Díaz Pita, "Los elementos característicos del dolo", ADPCP, 1990, p. 909 ss] у SCHROTH, Vorsatz als Aneignung der unrechtskonstituierenden Merkmale, Frankfurt a. M., 1994, pp. 115-118. Algo similar sucede en España con la partidaria de esta perspectiva, DíAz PITA, El dolo eventual, Valencia, 1994, p. 321. Una crítica al planteamiento de Schroth en SCHÜNEMANN, Hirsch-FS, pp. 368-369.

${ }^{10}$ En Alemania este punto de vista es defendido, en lo sustancial, por autores como FrIsCH, Vorsat\%, p. 95 ss; SCHMIDHÄUSER, "Die Grenze zwischen vorsätzlicher und fahrlässiger Straftat", JuS, 1980, p. 242; o JAKOBS, Strafrecht. Allgemeiner Teil, 2. ${ }^{a}$ ed., Berlín, 1993, p. 258 ss [trad. J Cuello y J.L. Serrano, Derecho penal. Parte general, Madrid, 1995, p. 316]. En España se defiende, entre otros, por Bacigalupo, Principios de Derecho penal, 5." ed., Madrid, 1998, p. 225; Silva SÁnchez, Aproximación, p. 401 ss; Gimbernat Ordeig, "Acerca del dolo eventual", en ID., Estudios de Derecho penal, 3. ${ }^{a}$ ed., Madrid, 1990, p. 259; LAURENZO Copello, Dolo y conocimiento, Valencia, 1999, p. 245; y FEIJóo SÁNCHEZ, "La distinción entre dolo e imprudencia en los delitos de resultado lesivo. Sobre la normativización del dolo”, CPC, 65 (1998), pp. 269 ss. Para una panorámica en
} 
Ragués - Consideraciones sobre la prueba del dolo

delitos de mera actividad es suficiente con que el sujeto sepa que en su comportamiento concurren aquellos elementos que integran el tipo objetivo penal. Una conclusión que, por lo demás, resulta plenamente coherente con la circunstancia de que aquello que excluya el dolo en los textos legales vigentes sea sólo el error y no una inexistente causa de eliminación de la voluntad.

Desde luego, esta perspectiva teórica no está exenta de problemas. Éstos se plantean, sobre todo, cuando sus partidarios tratan de determinar con precisión qué clase y grado de representaciones son necesarias para afirmar el nivel de conocimientos que exige una realización dolosa. Sin embargo, en sus puntos de partida no cabe duda de que esta teoría está mucho mejor encaminada que la primera, pues con ella se consigue un concepto válido tanto para delitos de resultado como para delitos de mera actividad y, además, es posible trazar una delimitación entre dolo e imprudencia que se corresponde con el merecimiento de pena propio de estas formas de imputación subjetiva sin por ello tener que recurrir a datos psíquicos de dudosa existencia práctica ${ }^{11}$.

Por todas estas razones, la definición del dolo como conocimiento de la realización típica se ha impuesto en la práctica del Derecho penal y ello pese a que una parte importante de la doctrina y, sobre todo, la jurisprudencia se resistan a abandonar la terminología propia de la teoría de la voluntad ${ }^{12}$. En la inmensa mayoría de ocasiones, quienes se declaran partidarios del dolo como intención acaban resolviendo los casos aplicando un dolo definido como conocimiento, de tal modo que, aunque en la doctrina parecen defenderse dos conceptos distintos, en realidad las discrepancias tienen sólo una naturaleza terminológica. Por consiguiente, la idea de dolo como conocimiento de los elementos del tipo objetivo es el enfoque del que se partirá en las siguientes páginas para analizar la cuestión de la prueba de dolo, una materia a la que, en adelante, se hará también referencia con la denominación de "prueba del conocimiento".

\section{LA PRUEBA DE LOS HECHOS SUBJETIVOS EN EL PROCESO}

Para construir una teoría completa del dolo no basta con definir este elemento del delito, sino que es necesario saber cómo debe constatarse en el proceso el dato fáctico del

\footnotetext{
lengua alemana del estado actual de doctrina y jurisprudencia españolas en materia de dolo cfr. RAMOS TAPIA, "Die Entwicklung des Vorsatzbegriffs in der spanischen Strafrechtswissenschaft", ZStW, 113 (2001), pp. 401418. En Argentina cabe citar a SANCINETTI, Teoría del delito y disvalor de la acción, Buenos Aires, 1991, pp. 201202, entre los partidarios del dolo como conocimiento.

11 En la doctrina actual pretende ir más allá JAKOBS, "Über die Behandlung von Wollensfehlern und von Wissensfehlern", ZStW, 101 (1989), pp. 530-531 [trad. C.J. Suárez, "Sobre el tratamiento de los defectos volitivos y de los defectos cognitivos" en JAKOBS, Estudios de Derecho penal, Madrid, 1997, pp. 128-146], quien considera que ciertos desconocimientos (provocados o debidos a la indiferencia del sujeto) merecen el mismo tratamiento que casos de dolo. Este punto de vista, próximo a la figura anglosajona de la willful blindness, se acoge en alguna sentencia del Tribunal Supremo español, como la STS de 16 de octubre de 2000 (ponente Giménez García), en la que se afirma que, "quien se pone en situación de ignorancia deliberada, sin querer saber aquello que puede y debe saberse, y sin embargo se beneficia de la situación -iba a cobrar un millón de ptas.- está asumiendo y aceptando todas las consecuencias del ilícito negocio en el que voluntariamente participa" (negrita en el original). El mismo razonamiento se plantea en la STS de 10 de enero de 2000 (ponente Giménez García).

12 Esta idea ya se expresaba en la Sentencia del Tribunal Supremo español de 23 de abril de 1992 (ponente Bacigalupo Zapater), en la que se reconocía que, bajo los "ropajes" terminológicos de la teoría del consentimiento, las decisiones de este Tribunal se hallaban cada vez más próximas a los postulados de la teoría de la probabilidad, que es una de las variantes más extendidas de la teoría de la representación.
} 
que depende su aplicación: los conocimientos del acusado en el momento de delinquir ${ }^{13}$. Lamentablemente, si uno trata de encontrar en la doctrina construcciones teóricas que analicen esta cuestión no hallará muchas respuestas pese a su indudable importancia para la aplicación cotidiana del Derecho Penal. La dogmática tradicional no ha manifestado un especial interés por analizar el traslado de los conceptos teóricos a la práctica forense, argumentando a menudo que ésta es una tarea que compete a la ciencia procesal. Sin embargo, los procesalistas tampoco se han mostrado muy dispuestos a desarrollar su teoría general de la prueba o de los medios probatorios pensando en los concretos elementos de la infracción punible, de tal modo que, hasta hace poco, apenas era posible encontrar trabajos que se ocuparan de esta materia ${ }^{14}$.

Esta situación no puede en modo alguno valorarse positivamente, pues la mutua ignorancia entre lo sustantivo y lo procesal hace imposible saber cuál es el exacto alcance de las teorías que la ciencia penal formula sobre el dolo. No en vano, con una aplicación procesal flexible conceptos muy estrictos de dolo pueden llevar a castigar frecuentemente en la práctica por delito doloso, mientras que conceptos teóricamente muy amplios pueden conducir a un menor número de condenas si en la práctica el juez es muy restrictivo en el momento de la prueba. En este sentido, debe afirmarse que las reglas de prueba desempeñan un importante papel político-criminal de delimitación del ámbito de lo punible y que, por tal razón, es muy necesario que la ciencia — ya sea procesal o penal— trate de teorizar sobre ellas ${ }^{15}$.

La demostración en el proceso penal del conocimiento o las representaciones de un acusado en el momento de realizar la conducta delictiva entra dentro de lo que jueces y tribunales suelen denominar la prueba de hechos subjetivos o psicológicos. Según se afirma, la constatación de estos hechos resulta especialmente compleja, pues, a diferencia de lo que sucede con la prueba de otros elementos fácticos, el conocimiento ajeno es un dato que se sitúa más allá de la percepción sensorial y, por tanto, para su descubrimiento bien poca cosa pueden aportar los medios probatorios más habituales, como la prueba testifical ${ }^{16}$. Semejantes dificultades no se circunscriben al dolo, sino que surgen también cuando se intenta constatar en el proceso la presencia de otros fenómenos de naturaleza psíquica,

\footnotetext{
13 En este sentido puede afirmarse que las actuales definiciones de dolo no pretenden una teoría completa sobre este concepto, algo que, por el contrario, no sucedía en teorías anteriores al siglo XIX, como la del dolus ex re o el dolus indirectus, que conjugaban las cuestiones probatorias con las conceptuales. Sobre estas figuras, cfr. LESCH, JA, 1997, p. 83; PuPPE, "Der Vorstellungsinhalt des dolus eventualis", ZStW, 103 (1991), p. 26 y VOLK, "Dolus ex re", en Arthur Kaufmann-FS, Heidelberg, 1993, p. 106 ss. En Italia, BricOlA, Dolus in re ipsa. Osservazzioni in tema di ogetto e di accertamento del dolo, Milán, 1960.

14 Algunas excepciones dignas de ser citadas son HRUSCHKA, "Über Schwierigkeiten mit dem Beweis des Vorsatzes", en Kleinknecht-FS, Múnich, 1985, p. 191 ss; FrEund, Normative Probleme der "Tatsachenfeststellung”, Heidelberg, 1987; FRISCH, "Gegenwartsprobleme des Vorsatzbegriff und der Vorsatzfeststellung - am Beispiel der AIDS-Diskussion", en K. Meyer-GS, Berlín, 1990, p. 533 ss; y VOLK, "Begriff und Beweis subjektiver Merkmale", en 50 Jahre Bundesgerichtsthof, vol. IV, Múnich, 2000, p. 739 ss. En España se ocupa de la cuestión DíEz Ripollés, Los elementos subjetivos del delito. Bases metodológicas, Valencia, 1989 y PÉREZ DEL VALLE, "La prueba del error en el proceso penal", RDP, 1994, p. 413 ss.

15 Se plantea la posibilidad teórica de integrar en los conceptos dogmáticos los recursos para su aplicación práctica, Bacigalupo Zapater, "Presunción de inocencia, 'in dubio pro reo' y recurso de casación", ADPCP, 1988, p. 379. Sobre la relación entre prueba y concepto en los elementos subjetivos cfr. VoLK, en 50 Jahre Bundesgerichtshof, pp. 746-747.

${ }^{16}$ Una perspectiva sobre cómo argumenta la jurisprudencia española en materia de dolo puede encontrarse en Laurenzo Copello, Dolo, pp. 152-177.
} 
Ragués - Consideraciones sobre la prueba del dolo

como aquéllos en que se basan los elementos subjetivos del tipo o el conocimiento de la antijuricidad ${ }^{17}$.

Tradicionalmente se ha entendido que, para la prueba de los hechos psíquicos, existen dos grandes medios probatorios. En primer lugar, la confesión autoinculpatoria, que, según suele afirmarse, es la prueba por excelencia de la existencia de dolo, puesto que sólo el acusado sabe realmente qué pasaba por su cabeza en el momento de cometer los hechos. Y, en segundo lugar, la prueba de indicios, es decir, la aplicación por parte del juez de determinadas máximas de experiencia a hechos de naturaleza objetiva previamente probados. Este segundo medio probatorio es el recurso al que más frecuentemente se acude en la práctica para atribuir conocimientos, ya que las confesiones autoinculpatorias no son demasiado frecuentes ${ }^{18}$.

No obstante, según la perspectiva tradicional el empleo de estos dos medios probatorios sólo sirve para acreditar un hecho cuando, como resultado de su valoración, se provoca en la persona del juez la intima convicción de que tal hecho ha sucedido realmente. El convencimiento judicial como culminación de la actividad probatoria ha ido ligado históricamente a los sistemas de libre valoración de la prueba que predominan en los ordenamientos procesales más modernos desde que cayeran en desgracia los sistemas de prueba tasada ${ }^{19}$. Sin embargo, la íntima convicción como objetivo de la actividad probatoria plantea un grave problema teórico: dado que las convicciones personales son algo muy variable de una persona a otra, si se acoge la convicción del juez como criterio decisivo para una correcta valoración de la prueba se hace depender la solución final de los casos de un factor subjetivo y cambiable, lo que supone aceptar, de forma más o menos explícita, que dos casos absolutamente idénticos pueden tener soluciones radicalmente distintas en función de quien los enjuicie y que, pese a ello, la solución de los dos casos deberá considerarse correcta ${ }^{20}$.

Aunque es evidente que en la práctica cotidiana inevitablemente casos iguales van a tener soluciones distintas, el que esto suceda no puede dejar de ser visto como algo anómalo, por lo menos si se pretende que la labor de la ciencia penal tenga algún sentido. Dicho en otras palabras, la ciencia penal y, en especial, la dogmática jurídica, sólo tienen razón de ser si se parte de la premisa teórica según la cual dos casos idénticos sólo pueden tener una solución correcta. Acogiendo este punto de vista debe afirmarse, por tanto, que no toda íntima convicción del juez por el mero hecho de producirse ya debe ser aceptada como

\footnotetext{
17 Sobre el tratamiento que la jurisprudencia del Tribunal Supremo español dispensa a la prueba del conocimiento de la antijuricidad, cfr. FELIP I SABORIT, Error iuris. El conocimiento de la antijuricidad y el art. 14 del Código Penal, Barcelona, 2000, pp. 163-206.

18 Con todo, hoy en día ya no se entiende que la confesión suponga la "prueba plena" de los elementos subjetivos, mientras que la prueba de indicios sería una prueba de segunda clase, sino que se atribuye a ambos el mismo rango en tanto que medios probatorios, dado que se admite la posibilidad de que la confesión no necesariamente concuerde con la realidad de lo sucedido. Cfr. al respecto HRUSCHKA, Kleinknecht-FS, p. 199, quien argumenta que, en el fondo, la confesión sólo es un indicio más.

19 Ésta es la concepción que justifica la redacción de artículos como el 741 de la Ley de Enjuiciamiento Criminal española o el $\ 261$ de la StPO alemana.

${ }^{20}$ En la doctrina ponen de manifiesto el carácter irracional del acto de convicción STEIN, “Gewißheit' und 'Wahrscheinlichkeit' im Strafverfahren”, en WOLTER (ed.), Zur Theorie und Systematik des Strafprozeßrechts, Neuwied, 1995, p. 256 y FrEund, Normative Probleme, prólogo, s/n. Por su parte HANACK, "Maßstäbe und Grenzen richterlicher Überzeugungsbildung im Strafprozeß”, JuS, 1977, p. 728, habla del carácter "subjetivo" del acto de convicción y NELL, Wabrscheinlichkeitsurteile in juristischen Entscheidungen, Berlín, 1983, pp. 105 y 107 del carácter "emocional".
} 
REJ - Revista de Estudios de la Justicia - No 4 - Año 2004

válida, sino que tal convicción sólo será aceptable si coincide con la solución correcta del caso que se está juzgando. Lógicamente, la cuestión que, llegados a este punto, debe resolver la ciencia penal es la de cuáles son los criterios para decidir en qué casos es correcta una convicción judicial.

Una respuesta sólo parcial a esta pregunta la ofrece la denominada "teoría de los dos niveles en la valoración de la prueba". Según esta perspectiva, en aquellos casos en que la apreciación de la prueba no depende necesariamente de la inmediación judicial, una determinada convicción no puede aceptarse sin más como correcta si su contenido se opone a los conocimientos científicos imperantes o contradice las reglas de la lógica y la experiencia $^{21}$. En la práctica, la implantación de esta teoría, por lo menos en la jurisprudencia española, ha tenido un efecto altamente positivo, pues con ella los tribunales superiores han admitido la posibilidad de revisar la valoración de la prueba realizada por los jueces o tribunales de instancia, una posibilidad que no cabía con las concepciones más tradicionales de la íntima convicción, que se asentaban en una visión absolutamente subjetiva de este requisito ${ }^{22}$.

No obstante, y pese al indudable avance que ha supuesto esta teoría, con ella se sigue planteando el problema de que, aunque consiguen rechazarse las conclusiones judiciales absurdas, se sigue admitiendo que dos casos idénticos pueden tener dos soluciones correctas distintas, siempre que ninguna de ellas pueda calificarse de ilógica o de científicamente insostenible, algo que, afortunadamente, pocas veces sucede en la práctica $^{23}$. Para superar este inconveniente es necesario construir una teoría que no se limite a rechazar aquellas convicciones que puedan calificarse de insostenibles, sino que, de entre las diversas convicciones "sostenibles" que puedan imaginarse para un caso concreto, establezca cuál debe acogerse como correcta.

\section{PROPUESTA DE TRATAMIENTO: LAS REGLAS DE ATRIBUCIÓN DEL CONOCIMIENTO}

En el caso de la "prueba del conocimiento" la elección de un criterio teórico que permita determinar la solución correcta exige analizar el contenido de las denominadas "reglas de experiencia" y, de forma más precisa, de aquéllas que pueden denominarse "reglas de experiencia sobre el conocimiento ajeno", que sirven para determinar, a partir de la concurrencia de ciertos datos externos, qué es lo que se representó una persona en el momento de llevar a cabo una determinada conducta. En consecuencia, la búsqueda de criterios para saber cuál es la decisión correcta en este ámbito no pasa por intentar evitar

\footnotetext{
21 Para conocer cómo aplica la jurisprudencia española esta teoría resulta fundamental la STS de 15 de abril de 1989 (ponente Bacigalupo Zapater). En Alemania, cfr. entre otras la sentencia BGH, NStZ, 1988, p. 236 ss; MAIWALD, AK-StPO, \261, n. ${ }^{\circ}$ marg. 13 ss; y SCHMIDT, Grundsätze der freien richterlichen Beweiswürdigung im Strafprozeßrecht, Frankfurt a.M., 1994, p. 90 ss.

22 De acuerdo con las perspectivas más tradicionales de la convicción judicial, ésta era siempre un acto "libérrimo" y, por tanto, irrecurrible. Cfr. en este sentido la STS de 10 de febrero de 1978 (ponente Vivas Marzal). En esta resolución se presenta esta libertad absoluta del juez en el momento de valorar la prueba como un avance frente al sistema - "felizmente superado", decía el Tribunal Supremo- de la prueba tasada. Sobre las raíces históricas de este razonamiento cfr. HOYER, "Der Konflikt zwischen richterlicher Beweiswürdigungsfreiheit und dem Prinzip 'in dubio pro reo", ZStW, 105 (1993), p. 527.

23 La posibilidad de esta doble solución correcta se admite de hecho en la doctrina por autores como MiRANDA ESTRAMPES, La minima actividad probatoria en el proceso penal, Barcelona, 1997, pp. 152-154.
} 
Ragués - Consideraciones sobre la prueba del dolo

que el juez llegue a conclusiones opuestas a las reglas de experiencia, sino de ver, en cada caso, cuál es la solución que dichas reglas imponen como correcta ${ }^{24}$.

Todo parece indicar que el parámetro para decidir sobre la corrección de una determinada regla de experiencia no puede ser otro que la existencia de amplio consenso social en torno a su vigencia. Semejante conclusión se justifica, ante todo, por la función social que el Derecho penal desempeña, una función que sólo tendrá consecuencias legitimables si los mensajes que la justicia penal dirige son aceptables y comprensibles desde el punto de vista los ciudadanos. Por este motivo debe afirmarse que la tarea del juez no debe consistir en construir o inventar las reglas de experiencia para cada caso concreto, sino en acudir a la interacción social para buscar dichas reglas. En el caso concreto de la prueba del dolo deberá aislar aquellas reglas que se emplean en sociedad para las atribuciones mutuas de conocimientos entre ciudadanos ${ }^{25}$.

Sólo cuando el juez encuentre en dicha interacción una regla de experiencia de vigencia indiscutible según la cual, presupuestos ciertos datos objetivos, una persona por fuerza ha sido conocedora de determinados hechos, podrá atribuir correctamente dichos conocimientos al concreto acusado. Cualquier convicción judicial que se aparte de este criterio deberá ser considerada arbitraria, una consideración que puede tener importantes efectos -sustantivos y procesales- en sistemas que, como el español, proclaman la interdicción de la arbitrariedad de los poderes públicos en su texto constitucional (art. 9.3 $\mathrm{CE}$ ), lo que permite afirmar que toda valoración arbitraria de la prueba supone la infracción de un precepto constitucional.

Las anteriores ideas sirven para resolver complejas cuestiones que pueden plantearse en la práctica, como, por ejemplo, la valoración que merecen ciertas opiniones periciales acerca de las hipotéticas representaciones de los acusados en el momento de suceder los hechos. Por mencionar un ejemplo concreto, en el caso de algunos homicidios pasionales se plantea en ocasiones por ciertos psicólogos o psiquiatras que posiblemente el acusado no haya sido plenamente consciente de cuál era la auténtica trascendencia de sus actos. De acuerdo con un planteamiento tradicional, las observaciones de estos peritos podrían llevar a negar el dolo en el caso de suscitarse una duda en la persona del juez ${ }^{26}$. Sin embargo, desde el punto de vista que aquí se defiende tales opiniones no tienen valor alguno para la constatación forense del conocimiento ajeno, de tal modo que, cuando la conducta realizada por el sujeto se considere como forzosamente consciente desde el punto de vista de la interacción social, ésta deberá ser también la conclusión del juez.

\footnotetext{
24 Algunos fundamentos para sustentar esta conclusión pueden encontrarse en trabajos tan diversos como, en Alemania, Stein, en Zur Theorie, p. 234; en Italia, Framarino Dei MALATESTA, Lógica de las prueba en materia criminal, 3. ${ }^{a}$ ed., Turín, 1912 (trad. S. Carrejo y G. Guerrero, 4. ${ }^{a}$ ed., Bogotá, 1995), p. 57; y, en España, IgaRTUA SalaVerRia, Valoración de la prueba, motivación y control en el proceso penal, Valencia, 1995, pp. 154-155.

${ }^{25}$ De forma más o menos explícita éste es el punto de vista que, para la constatación de los elementos subjetivos, parecen sustentar autores como HruschKA, Kleinknecht-FS, p. 191 ss; y Krauß, "Der psycologische Gehalt subjektiver Elemente im Strafrecht", Bruns-FS, Colonia, 1978, p. 22. Para un mayor desarrollo del punto de vista particular, véase RAGUÉS I VALLÈs, El dolo ..., op. cit., pp. 323-353.

${ }^{26}$ Cfr. al respecto diversos planteamientos recogidos en trabajos como PRITTWITZ, "Dolus eventualis und Affekt. Ein Beitrag zur Kriminologie des Allgemeinen Teils des StGB", GA, 1994, p. 454 ss; KARgL, Der strafrechtliche Vorsatz auf der Basis der kognitiven Handlungstheorie, Frankfurt a.M., 1993, p. 15 ss, así como la obra en la que, en buena medida, se basan estos dos autores: LEMPP, Jugendliche Mörder. Eine Darstellung an 80 vollendeten und versucbten Tötungsdelikten von Jugendlichen und Heranwachsenden, Berna, 1977, p. 20.
} 
Tal negativa se explica porque, desde la perspectiva de las actuales valoraciones sociales, a los psiquiatras y psicólogos tal vez se les reconozca competencia para aportar información sobre cuándo un sujeto padece una determinada anomalía psíquica o enfermedad mental, pero no para descubrir qué es lo que dicho sujeto sabía o se representó en el momento ya pretérito en que llevó a cabo una conducta objetivamente típica. Esta conclusión, que hasta cierto punto puede sorprender con respecto a los delitos de sangre, parece casi una obviedad en delitos de naturaleza económica o patrimonial, en los que resulta evidente que nada pueden aportar psicólogos o psiquiatras en relación con la prueba del conocimiento de un acusado ${ }^{27}$.

La anterior afirmación tiene varias repercusiones, unas de orden sustantivo y otras de orden procesal. La primera de ellas es poner claramente de manifiesto que en el proceso penal el juez no puede echar mano sin más de cualquier medio probatorio imaginable para la prueba de cualquier elemento del delito, sino que los medios probatorios admisibles deben variar en función de lo que deba ser probado. En este ámbito el criterio decisivo debe ser el grado de confianza social en la certeza de los resultados de cada medio, lo que permite sostener, por ejemplo, que para la "prueba del dolo" o de otros requisitos de la infracción penal, como los elementos subjetivos del tipo o el conocimiento de la antijuricidad, el juez no puede ni debe acudir a la prueba pericial, porque actualmente no existe suficiente confianza en los resultados de dicho medio probatorio. En cambio, estas periciales se convierten en el recurso por excelencia cuando se trata de determinar la existencia de determinados transtornos psíquicos en la persona del acusado ${ }^{28}$.

Desde un punto de vista sustantivo las anteriores ideas permiten observar también cómo el modelo de sujeto con el que trabaja la teoría del delito varía según el escalón de dicha teoría en el que se opera. Tal afirmación resulta evidente si el modelo de hombre empleado en la imputación objetiva se compara con el utilizado en la culpabilidad, pues mientras en el primer nivel se razona mayoritariamente con el denominado "hombre medio" o, mejor dicho, el "hombre perfecto", que advierte y prevé todos los peligros relevantes, en la culpabilidad se tiene en cuenta al acusado individualmente considerado, con todas sus anomalías y limitaciones personales. A diferencia de lo que suele presuponerse, en el ámbito del dolo (y de su prueba) no se opera propiamente con ninguna de estas dos figuras, sino con un tercer modelo resultante de contextualizar al hombre medio en el escenario del concreto acusado, añadiéndole buena parte de sus características personales, pero sin tener en cuenta todavía determinados factores de su individualidad, como, por ejemplo, las enfermedades psíquicas que pueda padecer, el grado de impregnación alcohólica que presentaba en el

\footnotetext{
27 Este punto de vista coincide con lo manifestado por KRAUB, Bruns-FS, p. 593 o LoOS, "Grenzen der Umsetzung der Strafrechtsdogmatik in der Praxis”, en Rechtswissenschaft und Rechtsentwicklung, Göttingen, 1980, p. 272, quien sostiene que las ciencias empíricas carecen del instrumental necesario para constatar procesos psíquicos singulares e irrepetibles. Una mayor consideración hacia el papel que pueden desempeñar los peritos en la prueba de los elementos subjetivos en DíEz RipolLés, Los elementos subjetivos, pp. 306-307.

${ }_{28}$ Claramente contradictorio lo sostenido en la STS de 30 de marzo de 1999 (ponente Martínez Arrieta), referida a un caso de prueba del ánimo lúbrico en un abuso sexual. Aunque en un párrafo se afirma tajantemente que los elementos subjetivos "se sustraen a las pruebas testificales y periciales en sentido estricto", en el párrafo siguiente se afirma la existencia de ánimo lúbrico apoyándose explícitamente en "criterios suministrados por los peritos". Con todo, esta sentencia es una excepción dentro de la jurisprudencia española sobre elementos subjetivos, pues en la inmensa mayoría de sentencias no se plantea siquiera la posibilidad de acudir a la prueba pericial, casi siempre porque las partes tampoco han solicitado su práctica en juicio.
} 
Ragués - Consideraciones sobre la prueba del dolo

momento de suceder los hechos o el estado de arrebato u obcecación en que se hallaba cuando delinquió 29 .

Sólo razonando de este modo se impide una doble atenuación injustificada -por falta de dolo y de plena imputabilidad- en el caso de ciertas anomalías psíquicas o intoxicaciones, evitándose asimismo tratar como imprudentes conductas que en las valoraciones cotidianas nadie juzgaría como tales ${ }^{30}$. Este es el caso, por ejemplo, del sujeto que, obcecado porque su pareja sentimental le abandona por otro, provoca su muerte propinándole con una plancha numerosos golpes en la cabeza ${ }^{31}$. El que en casos como éste psicólogos o psiquiatras puedan argumentar que el grado de obcecación fue tal que el sujeto no era verdaderamente consciente de la trascendencia de sus actos no impide afirmar que existe un homicidio doloso, pues para tal conclusión lo único que importa es la vigencia de una regla de experiencia social según la cual quien propina a otra persona más de cincuenta golpes en la cabeza con una plancha de hierro es consciente de que puede ocasionar su muerte. Por supuesto, la situación personal del concreto acusado deberá ser tenida en cuenta en el momento de analizar su culpabilidad, pero no antes ${ }^{32}$.

Con afirmaciones de este tipo se asume de forma explícita un evidente riesgo de que las conclusiones del juez acerca del conocimiento del acusado puedan no coincidir con la realidad de aquello que dicho acusado verdaderamente supo o se representó en el momento de los hechos ${ }^{33}$. Aunque semejante constatación pueda escandalizar, no debe perderse de vista que este riesgo no es algo exclusivo de la prueba del dolo según el planteamiento aquí defendido, sino que es un riesgo inherente a toda condena dictada en la jurisdicción penal, pues, no en vano, y como afirma algún autor, si se llevara hasta el último extremo la idea del in dubio pro reo jamás debería condenarse, aunque sólo fuera por la duda sobre si las verdades que hoy se asumen como tales dejarán de serlo con el paso de los años ${ }^{34}$.

\footnotetext{
29 La idea de que en el ámbito de la prueba de los elementos subjetivos nunca se pretende una plena subjetivización del acusado es puesta de manifiesto por VOLK, en 50 Jahre Bundesgerichtshof, pp. 752-753. Sostiene este autor (ibidem, p. 752) que en estos razonamientos siempre se acude a lo que "uno' habría pensado, visto, sentido o creído en esta situación concreta".

${ }^{30}$ En estos términos razona implícitamente Roxin, Strafrecht. Allgemeiner Teil, vol. I, 3. ${ }^{a}$ ed., Múnich,1997, \ 12, n. ${ }^{\circ}$ marg. 117, quien afirma que las conductas realizadas en estados pasionales no guardan ningún parecido con las conductas imprudentes, entendidas éstas como descuidos.

${ }^{31}$ Caso citado por SCHILD, "Strafrechtsdogmatische Probleme der Tötung des Intimpartners", JA, 1991, p. 48 ss.

32 En un mismo sentido PupPe, $N K_{s} \ 15$ n $^{\circ} .^{\circ}$ marg. 131 ss. Aunque sin explicitar los fundamentos teóricos que lo justifican, éste es también el planteamiento del Tribunal Supremo español (cfr., por ejemplo, la STS de 13 de noviembre de 1991 [ponente Montero Fernández-Cid]), que (casi) siempre ha negado que circunstancias como la obcecación, embriaguez o alteraciones psíquicas puedan ser tenidas en cuenta para negar el dolo del acusado. Excepcionalmente, no obstante, en alguna resolución se toman en cuenta los transtornos de personalidad como indicio para negar que un sujeto actuara con dolo; cfr., por ejemplo, la STS (Sala 5. ${ }^{a}$ ) de 15 de marzo de 1997 (ponente Aparicio Gallego).

${ }^{33}$ Con una franqueza fuera de lo común, esta posibilidad es reconocida por la STS de 14 de julio de 1989 (ponente García Miguel), en la que se afirma que "razones de seguridad jurídica colectiva, en tanto en cuanto el adelanto de la ciencia no permita un mayor conocimiento de lo que pasa en la mente de una persona" obligan a acudir a otros criterios "aun a sabiendas de lo erróneo y, en consecuencia arbitrario, que en ocasiones puedan resultar los juicio de valor que se formulen".

34 Una idea planteada en estos términos por FrEUND, Normative Probleme, p. 6. En términos similares ya Framarino Dei MaLATESTA, Lógica, p. 86, quien reconoce que "lo que falsamente aparece como increíble ante la ignorancia de una generación, puede presentarse exactamente como creíble a los conocimientos de la generación posterior".
} 
Una dogmática penal que quiera hacerse verdaderamente merecedora de la consideración de ciencia no puede ignorar semejante riesgo, ni puede, para eliminarlo aparentemente, legitimar puros subjetivismos como la convicción judicial. Como afirmaba el procesalista italiano Framarino dei Malatesta, hablar de certeza allí donde sólo hay una probabilidad sólo sirve para encontrar un "cómodo sillón a la pereza que incita a descansar en él con el pretexto hipócrita de una buena conciencia"35. Para superar esta tentación, la dogmática debe, por un lado, intentar reducir al máximo dicho riesgo de error dentro de los márgenes que permite la ineludible necesidad de pena que suscitan determinados hechos y, por otro lado, debe procurar criterios racionales y uniformes que eliminen la sensación de que las soluciones dependen de un acto puramente emocional del juez. La reducción del riesgo de error a unos niveles que permitan considerar legítima su asunción, así como búsqueda de racionalidad y uniformidad en las soluciones son los dos grandes objetivos que deberán guiar la construcción de una nueva teoría de la prueba pensada para la aplicación procesal de los diversos elementos conceptuales que conforman la infracción penal ${ }^{36}$.

Para mostrar con un ejemplo cómo puede llevarse a cabo esta necesaria labor, seguidamente se analizará el complejo problema de la prueba del dolo del resultado en aquellos delitos que, como el homicidio o las lesiones, exigen este elemento típico. En este análisis se aislarán algunos criterios que permitan alcanzar los dos objetivos trazados: máxima reducción, en la medida de lo posible, del riesgo de error y uniformidad en el tratamiento de los casos.

\section{LA PRUEBA DEL DOLO EN LOS DELITOS DE RESULTADO}

En el caso de los delitos de resultado la doctrina y jurisprudencia mayoritarias entienden que, para poder hablarse de una realización dolosa, es necesario que el acusado se haya representado el riesgo concreto de producción del resultado típico que creaba con su conducta ${ }^{37}$. En la práctica es bastante frecuente encontrar casos en que puede atribuirse al acusado el conocimiento en abstracto del riesgo que generaba su comportamiento, así como un conocimiento correcto de las circunstancias fácticas que lo rodearon, pese a lo cual su defensa alega en el juicio que su cliente no integró estos dos conocimientos en el juicio de apreciación del concreto riesgo que exige el dolo de los delitos de resultado ${ }^{38}$. Piénsese, por ejemplo, en el sujeto que, intentando huir de la policía después de un atraco bancario, dispara contra un agente hiriéndole mortalmente y que alega en juicio no haberse representado un riesgo concreto de muerte. $\mathrm{O}$ imagínese la misma alegación en el caso del conductor con prisas que decide saltarse un semáforo en rojo pensando que evitará a los otros vehículos y que acaba ocasionando una colisión mortal.

\footnotetext{
35 Framarino dei Malatesta, Lógica, p. 43.

36 Un intento de reducción del riesgo de error a unos límites que permitan legitimarlo teóricamente puede encontrarse en los trabajos de FrEUND, Normative Probleme, p. 58, entre otras; HOYER, ZStW, 105 (1993), p. 523 ss; y STEIN, en Zur Theorie, p. 233 ss.

${ }^{37} \mathrm{La}$ distinción entre conocimiento en abstracto del peligro — que da lugar a imprudencia— y conocimiento en concreto - que da lugar a dolo- es acogida en diversas resoluciones del Tribunal Supremo español, como, entre otras, la STS de 5 de febrero de 1997 (ponente Martínez-Pereda Rodríguez).

38 Para esta cuestión en general cfr. RAGUÉS I VALLÈs, El dolo, pp. 455-508.
} 
Ragués - Consideraciones sobre la prueba del dolo

En ambos casos el juez se encuentra ante sujetos que, en primer lugar, conocen en abstracto cuáles son los riesgos que crean con su conducta, pues, de acuerdo con la experiencia social, cualquier persona adulta en su sano juicio sabe que disparar contra otro crea un riesgo de muerte y lo mismo puede afirmarse de quien ignora un semáforo en rojo en la vía pública. Del mismo modo, en ambos casos puede atribuirse a los dos sujetos el conocimiento de las circunstancias en que actuaban: el atracador sabe que la pistola está cargada y que el agente se encuentra en las posibles trayectorias de la bala, del mismo modo que el conductor es consciente de que, si cruza en rojo, es posible que su vehículo colisione con los que se avecinan por la vía perpendicular. ¿Qué decisión debe tomar el juez ante las alegaciones de que estos conocimientos singulares no se integraron en el juicio de peligro concreto que exige el dolo?

A la anterior pregunta respondería la doctrina tradicional afirmando que la solución depende de la íntima convicción del juez a la vista de las pruebas practicadas en el acto del juicio. Sin embargo, por los motivos expuestos en páginas anteriores semejante planteamiento no puede aceptarse, sino que debe ser superado mediante la construcción de una teoría que permita establecer en estos casos cuál es la solución correcta de acuerdo con criterios racionales y válidos para cualquier otro supuesto similar. Por razones antes expuestas, para encontrar tal solución es necesario acudir a las reglas sociales de experiencia que rigen en materia de atribución del conocimiento ajeno.

Un análisis de dichas reglas permite afirmar que la experiencia social distingue, en lo que respecta a los riesgos que conllevan determinados comportamientos, entre conductas especialmente aptas para ocasionar ciertos resultados y conductas que, si bien son objetivamente capaces de provocar determinadas consecuencias lesivas, en la valoración social no están vinculadas indefectiblemente a su acaecimiento. La distinción entre conductas especialmente aptas y este segundo grupos de conductas — que en adelante serán denominadas "conductas neutras" - debe ser el criterio rector en la práctica para decidir cuándo una alegación de desconocimiento del riesgo concreto deberá ser creída.

En esta distinción influyen cuestiones muy diversas, como la utilidad social de determinadas actividades, la habituación que existe a ellas o la frecuencia estadística con la que su ejecución lleva al acaecimiento del resultado. En el caso del homicidio, por ejemplo, pueden citarse como especialmente aptas para causar una muerte conductas como disparar contra el cuerpo de otra persona o hacer explosionar una potente bomba en un lugar concurrido. En cambio, otros comportamientos como conducir un automóvil son sólo neutros en relación con el resultado, pues, aunque objetivamente pueden ocasionar una muerte, en la experiencia social esta consecuencia no es algo indisociablemente ligado a su realización.

En el caso en que el acusado haya realizado una conducta especialmente apta no deberá prosperar ninguna alegación por su parte en el sentido de haber desconocido en concreto el riesgo que estaba generando y, consecuentemente, se le deberá atribuir a título de dolo la causación del resultado correspondiente. En cambio, dicha alegación sí será creíble en el caso de las conductas neutras, debiendo imputarse sólo a título de imprudencia la causación del resultado típico. Así, por ejemplo, en el caso de quien provocó un accidente de tráfico y afirma no haberse representado en concreto el riesgo concreto de muerte que estaba creando, el juez está obligado a creer dicha alegación y sólo podrá condenar por imprudencia, mientras que, por el contrario, la misma alegación 
no deberá merecer la más mínima credibilidad si lo que hizo el sujeto fue provocar el estallido de un potente explosivo.

No obstante lo anterior, la afirmación de que en las conductas neutras es en principio creíble una ausencia de representación del riesgo que se estaba creando en concreto debe estar sujeta a algunas excepciones. Así, por ejemplo, en los casos en que el sujeto exteriorice de algún modo que sí es conocedor de dicho riesgo, en los supuestos en que la proximidad del acaecimiento del resultado se perciba mediante signos externos durante la realización de la conducta típica y, por último, cuando la dinámica comisiva no haga creíble -especialmente en los casos de minuciosa preparación- que el sujeto no haya recapacitado sobre los riesgos de su actuación, deberá atribuirse el conocimiento que exige el dolo de los delitos de resultado aun habiéndose realizado una conducta que, en abstracto, puede calificarse de "neutra".

Desde luego, a la presente construcción teórica se le puede criticar el que maneje conceptos cuya vaguedad hace difícil una aplicación inequívoca en el caso concreto. No obstante, y aun cuando esta crítica tenga cierta parte de razón, no puede ignorarse que también puede dirigirse la misma objeción a muchas otras teorías referentes a diversos elementos del delito, sin que por ello se declare el fracaso de tales teorías: conceptos como "previsibilidad" en la imputación objetiva, "fase ejecutiva" en el delito intentado o "posición de garante" en los delitos de omisión no son precisamente conceptos mucho más concretos que los que aquí se proponen para resolver la cuestión del dolo del resultado y, sin embargo, nadie reclama su abandono, sino que, en todo caso, lo que se persigue es un mayor grado de concreción teórica que haga posible un empleo menos arbitrario en la práctica.

Por otra parte, la solución propuesta tiene la gran ventaja de que permite discriminar qué indicios objetivos merecen ser tenidos en cuenta por el juez en el momento de resolver la cuestión de la prueba del dolo. Mientras los planteamientos tradicionales se conforman con una valoración de cualquier indicio imaginable con tal que el juez llegue a formarse una convicción, con la presente teoría se descartan determinados indicios utilizados a menudo en la práctica que, sin embargo, no parecen estar en condiciones de llevar a conclusiones aceptables cuando se trata de atribuir conocimientos a terceras personas sobre la base de la experiencia social. Éste es el caso, por ejemplo, de datos como la personalidad (violenta) del acusado, sus experiencias previas afortunadas en la realización de hechos similares, su arrepentimiento o frialdad posteriores a la conducta típica, la existencia o no de motivos para cometer el delito etc. ${ }^{39}$. Todos esos indicios frecuentemente valorados por los tribunales españoles para "probar" el dolo- tienen una naturaleza totalmente equívoca en relación con las representaciones del acusado en el momento de cometer el delito y, por tal razón, no deben ser tenidos en cuenta por el juez.

\footnotetext{
39 Todos estos "indicios" han sido tomados en consideración por el Tribunal Supremo español en algunas de sus sentencias: así, por ejemplo, en la STS de 5 de diciembre de 1991 (ponente Soto Nieto) se tiene en cuenta como indicio la "personalidad del agresor" y la del "agredido"; las experiencias previas afortunadas en la STS de 24 de noviembre de 1995 (ponente Delgado García); la actitud posterior al hecho del acusado en la STS de 1 de diciembre de 1991 (ponente Moyna Ménguez). Por estas mismas razones no puede asumirse tampoco la "Hemmschwellentheorie" que emplea a menudo la jurisprudencia del Tribunal Supremo alemán para negar el dolo homicida en aquellos casos en que no se encuentra fácilmente un motivo por el que se haya causado la muerte de la víctima. Una crítica a esta teoría en PUPPE, "Die Logik der Hemmschwellentheorie des BGH", NStZ, 1992, p. 576 ss y RoxIN, Strafrecht, $\int 12$, n. ${ }^{\circ}$ marg. 74 , con referencias.
} 
Ragués - Consideraciones sobre la prueba del dolo

Por último, otro gran avance del presente planteamiento es aportar una mayor previsibilidad en la resolución de los casos. Tal previsibilidad muy difícilmente puede conseguirse si se parte de que el criterio rector es la mera convicción. Sin embargo, cuando se entiende que sólo una solución puede ser correcta, el hecho de que un tribunal haya declarado que un determinado medio comisivo es especialmente apto deberá vincularle en sus posteriores resoluciones tanto como pueden hacerlo otras decisiones en materia de interpretación de los textos legales. A fin de cuentas, tan determinante es un extremo como el otro a efectos de determinar la punibilidad de ciertas conductas. Por esta vía debe evitarse que supuestos idénticos desde el punto de vista objetivo se acaben tratando de forma dispar en el ámbito de la tipicidad subjetiva en función de cuáles sean las apreciaciones o intuiciones personales de cada juez. Como se ha dicho antes, la pretensión de que lo igual se trate de igual manera no puede limitarse a la aplicación del Derecho penal sustantivo, sino que debe extenderse también a la valoración de la prueba. Hasta que esto no suceda, la dogmática penal sólo estará alcanzando a medias sus objetivos.

\author{
Abreviaturas empleadas: \\ S: parágrafo \\ ADPCP: Anuario de Derecho Penal y Ciencias Penales \\ AK-StPO: Kommentar zur Strafprozeßordnung (Reihe Alternativkommentare) \\ BGH: Bundesgerichtshof (Tribunal Supremo Federal de Alemania) \\ BGHSt: Repertorio jurisprudencial del BGH en asuntos penales (colección oficial) \\ CE: Constitución Española \\ CPC: Cuadernos de Política Criminal \\ FS: Festchrift für (Libro Homenaje a) \\ $G A$ : Goltdammer's Archiv für Strafrecht \\ GS: Gedächtnisschrift für (Libro en Memoria de) \\ $J A$ : Juristische Arbeitsblätter \\ $J u S$ : Juristische Schulung \\ $J Z$ : Juristenzeitung \\ NK: Nomos Kommentar zum Strafgesetzbuch \\ NStZ: Neue Zeitschrift für Strafrecht \\ RDP: Revista de Derecho Procesal \\ StGB: Strafgesetzbuch (Código penal alemán) \\ StPO: Strafprozeßordnung (Ordenanza Procesal alemana) \\ STS: sentencia del Tribunal Supremo español \\ ZStW: Zeitschrift für die gesamte Strafrechtswissenschaft.
}

